\title{
Data Mining Based Marketing Decision Support System Using Hybrid Machine Learning Algorithm
}

\author{
Dr. T. Senthil Kumar, \\ Associate Professor, \\ Computer Science and Engineering Department, \\ Amrita School of Engineering, \\ Coimbatore, TamilNadu, India. Email: \\ t senthilkumar@cb.amrita.edu
}

\begin{abstract}
Data mining is widely used in engineering and science, On the contrary, it is used in finance and marketing applications to resolve the challenges in the respective fields. Data mining based decision support system enhances the organization performance by analysing the ground reality. Turbulent economy is common for every organization due to the competition, cost, tax pressures, etc., Privatization, Globalization and liberalization drags the organization more into a competitive environment. In order to balance the competition and withstand to achieve desired gain proper marketing strategies are need to planned and executed. Marketing decision support system helps to reduce the organization burdens in analysing and strategical planning through its efficient data mining approach. This research work proposed a data mining based decision support system using decision tree and artificial neural network as a hybrid approach to estimate the marketing strategies for an organization.
\end{abstract}

Keywords: Data Mining, Marketing decision support system, Decision Tree, Artificial Neural Network.

\section{Introduction}

Due to the technology development, computer based business decision is commonly adopted even in small scale organizations to obtain a clear picture about the organization in next few years. Based on the analysis, the market value of an organization increases which provides more benefit to the owners and stack holders. Decision support systems are evolved to reduce the human burden in analysing the organizational data. It provides quick decisions based on the available information which could help to improve the organization quality with in short duration. Since, data quality defines the quality of an organization, so that decision could be taken at right time which diverts the growth towards right path. Decision support systems can able to select the data and analyse to obtain the right trends, so that strategies and solutions could be framed. Data is the main element in decision process so that the support system summarizes the information either graphically or textually using its expert artificial intelligence. Decision support systems collects the typical information such as assets, legacy and relational data and prepares comparative analysis as assumptions of future trend in a specific context. Most of the organizations adopted this decision support system as a day to day activity.

The common practice followed by an organization to collect the sales data at regular interval of time along with budget and forecast details in order to update the marketing strategy. Similarly, decision support systems collect information, analyse and reorganize the data to obtain constructive strategical decisions. Figure 1 depicts the general illustration of decision support system. 
Vol.02/ No. 03

Pages: $185-193$

http://irojournals.com/aicn/

DOI: https://doi.org/10.36548/jaicn.2020.3.007

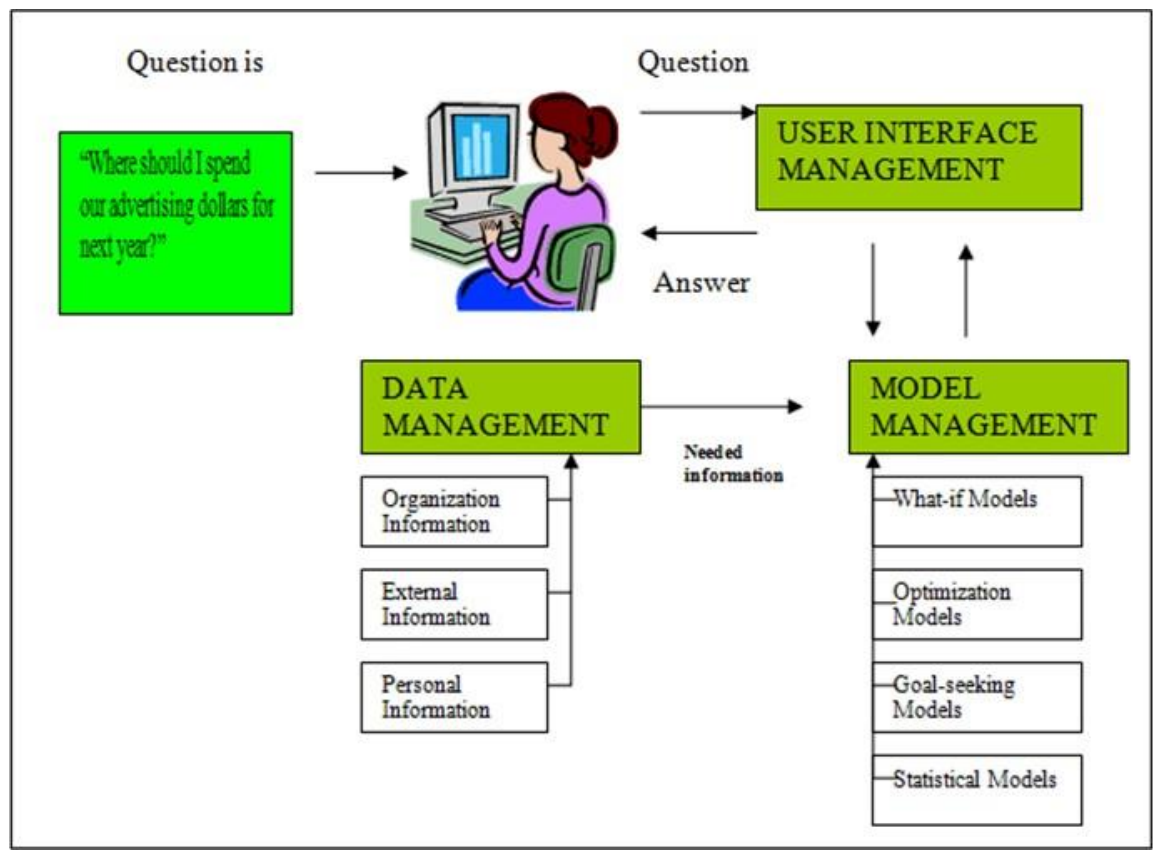

Fig. 1 Decision support system

Generally, decision support systems are differentiated into active and passive models. In which passive models collects the information and organize them in an effective manner and it do not provide any suggestions or decisions from the collected information. In case of active decision support system, it collects and process the information to produce solutions and strategies. On the other, cooperative decision support collects and analyse the data and along with human interaction it refines the solutions to obtain best strategies. Based upon the applications, decision support system is categorized into

- Data Driven Decision support system,

- Communication driven decision support system,

- Document driven decision support system,

- Model driven decision support system

- Knowledge driven decision support system.

Data driven decision support system concentrates on data collection process initially, since collecting essential information helps to obtain better decision with in short duration. Once the data is collected, it is manipulated based on the user needs, since the data may be of structured or unstructured due to internal and external sources it is essential to manipulate the data. A time series is framed to manipulate the internal data and if needed for external data also. Data driven decision support system provides simple file access system which could be accessed and retrieved as queries. Managers, employees, product suppliers and service persons are the target persons in data driven model to collect essential information. In case of communication driven decision support system analyses the shared tasks where more than a person is working on a single process. in order to obtain better strategy different collaborator are allocated to work together in communication driven models. Internal teams, partners are the main targets of communication driven model. Web based or client server based technology is widely adapted as communication driven process which provides decision support.

Document driven decision support systems performs operation based on group of users. Searching web pages and identifying relevant documents for a given specific set is the process in document driven models. Retrieval and analysis of documents is depending on storage and technology adaptation in this models. Variety of document types such as text document, database records and spreadsheets, etc., are processed to obtain decision in document driven models. Similar to communication driven, document driven decision support system also uses web based or client server based technology to obtain decision. Model driven decision support systems has option to 
manipulate the financial data to obtain optimized solution for organization growth. Generally, model driven decision support system does not require large dataset since it performs quantitative analysis and provides elementary functionalities using limited parameters. People interact with an organization, managers, staffs are the main targets of model driven decision support systems which could be executed in a client server or standalone personal computers.

Knowledge driven decision support systems has wide range of user coverage in an organization which includes others also to interact. Management advice is an essential process in knowledge driven models so that the organization could select the right service or products. It can also suggest actions to the managers. Knowledge driven model are like personal computers with problem solving skills so that understanding the problem in that domain could be identified easily. Benefits of decision support system is depicted in figure 2 along with its limitations.

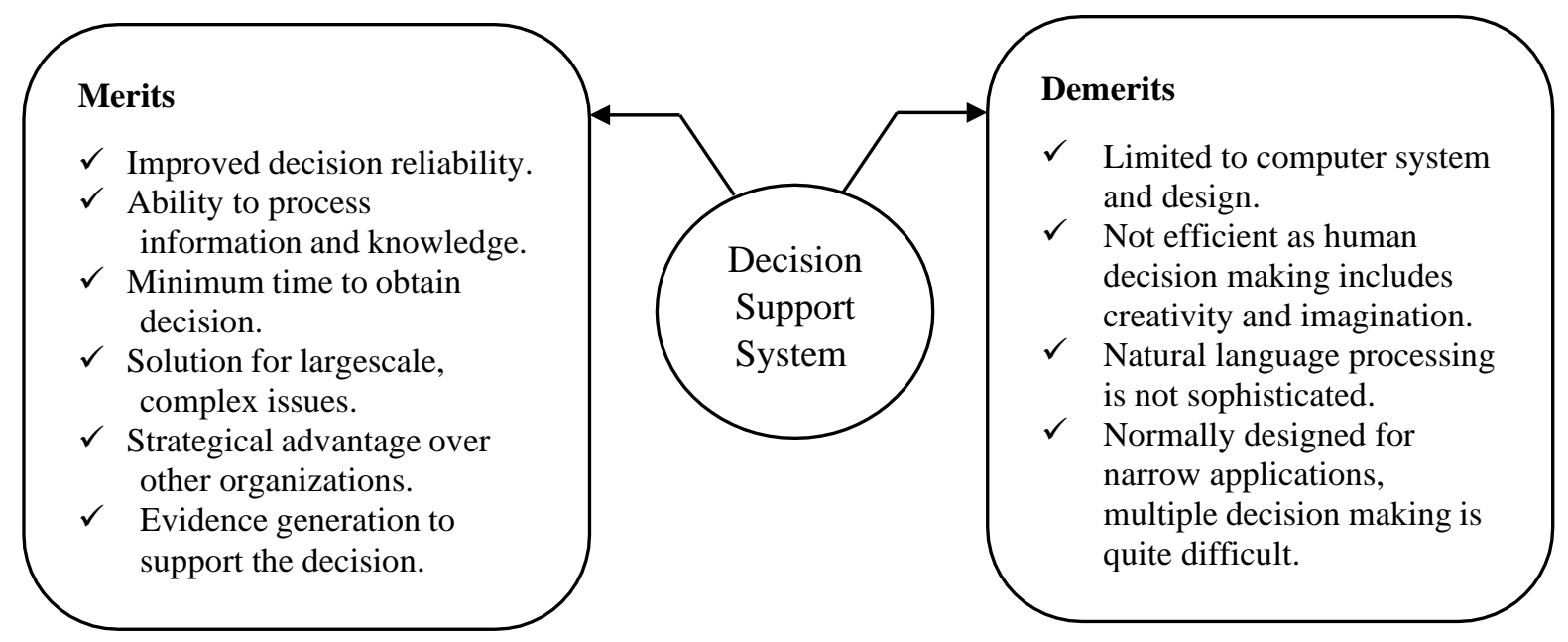

Fig. 2 Merits and Demerits of Decision support system

In other ways decision support systems employed in agriculture, where the farmers use this decision support for crop planning. Based on analysis, it provides suitable cultivating time, fertilize and harvest time which helps the farmers to plan accordingly. In case of medical field, decision support system is used as clinical decision support system which is used to manage the complex clinical data. Since medical data has wide range of versatile information such as tracking orders, preventive protocols, these decision models are employed as cost control models which reduces the repetitive, duplicate tests and drug purchase. Decision support system is used to predict the natural disasters like floods based on analysing the real time weather data and historical data. So that possible flood regions could be identified which avoid huge property loss and human causalities. Decision support system in educational institutions helps to obtain details about student details. Based on prediction it could define the students count for the upcoming year. Depends upon the results, institutions plan and process the student enrolment process to obtain full strength to run a course.

\section{Related works}

A vast survey has been made to obtain prior knowledge on issues in decision support system. based on application domain, system design and complexity, existing models are analysed and presented in this section. Daniel.et.al. [1] discussed about smart maintenance decision support system to manage corporate data. The research work brought insights on big data analytics and its decision making process. using a cost minimization algorithm, the proposed work discussed the issues in industry large scale data. Research work attains better predictive results for the case studies using its analytical decision process. In order to reduce faults in the production systems, knowledge and experience are the key factors. Sebastian.et.al. [2] considered those key 
factors in research work to obtain decision for distributed production environment. Since knowledge sharing is an important process for test and service teams which is directly related to quality of an organization, research work provides better decision support system using test cases. Incorporating test cases with fault symptoms the maintainers could able to identify the faults. İhsan Erozan.et.al. [3] proposed a decision support system for manufacturing industries to manage the activities. Parameters such as utilization rate, duty cycle, redundancy, stock effects are considered to define the proposed work which improves the maintenance performance and product reliability. In order to maintain critical components activities proposed decision support system is tested with industrial datasets which obtains better reliability compared to conventional decision models.

Francesco.et.al. [4] reported the issues in group decision support systems through k-means algorithm. Combined with hierarchical process research work attains remarkable decisions for the given tasks. In order to obtain the decision, alternatives are sorted and ordered based on its importance and Bezier curve fitting is used to obtain the decision makers preference function. The knapsack model used in this research work chooses alternatives based on the classes and budget constrains which yields better performance. Similarly, web based group decision support system is reported in Siddiqui.et.al. [5] research model. Research work is carried out to obtain solution for team preparation issue in a business school. Considering the course offerings, stakeholder information, assignments, timetable preparation, the observations are projected as multi stage issues. Proposed model used web application to define the workflow and automates the timetabling task using optimization model. Conventional spread sheet based tools could be replaced with improved quality and efficient workflow are the advantages of this decision support system.

Morteza.et.al. [6] considered the stability, delivery status, service attempts and financial growth to define the decision making system. To obtain the similarity and deployment of quality function these decision making is developed in the research work. Agricultural partners and investors are the target factors of this group decision making process. proposed research model provides an integrated and efficient decision making system which improves the reliability and quality while obtaining decisions. Katia.et.al. [7] reported the issues in agricultural production through decision support system as industrial processing based on the environmental, social and economic dimensions. Proposed model used evaluation mechanism to define the efficiency, cost and production factors. Sustainability index and semaphore are used to maintain the sustainability in the proposed approach.

Irrigation decision making and its related issues are reported in Nicholas.et.al. [8] research work. Heed decision theory and decision modelling is proposed in the research work to distinguish the decision support system tasks and real time decisions. In order to process and obtain business solutions decision modelling notation is suggested, decision ontology is suggested for web modelling and decision modelling ontology is suggested for conventional ontology. Yang Luo.et.al. [9] reported the issues in data centre and its decision support system to evaluate the energy and temporal availability. Proposed work is framed to analyse the issues in medium sized commercial data centre using four stage framework. Considering the cost benefits, environmental impact. Proposed decision support system is designed to deliver an optimized heat recovery process. Through proposed approach the overall energy requirement of data centre is streamlined.

Rule based reasoning is proposed in Mahsa.et.al. [10] research work along with fuzzy logic classifier to obtain the triage level of patients. These clinical decision support system provides prediction results based on emergency service index which has patient vital signs. These vital signs are considered as inputs to fuzzy logic to predict the triage levels. Similar clinical decision support system is reported in Ahmed.et.al. [11] research work which uses 1means clustering for decision making process. Processing the leukemia cells, the features are extracted to obtain decision based on the internal structure. Proposed work is experimentally verified using public dataset which effectively identifies the leukemia cells compared to conventional clinical decision support systems. Another clinical decision support system is reported by Marieke.et.al. [12] to measure the plasma concentration in therapy. By measuring the plasma concentration, patient health condition is efficiently reviewed using proposed decision support system.

Muhammad.et.al. [13] reported the issues in rubber production through decision support system to identify the yields in a smallholder rubber production unit. Research model considers the institutional factors and social impacts on rubber production through decision support system which improves the profitability and viability of the farm management. Zouhair.et.al. [14] proposed a proactive decision support system to predict the crash events on vehicle kinematics. Considering the roadway geometrics, driver inputs and real time weather data proposed 
decision support system is developed through machine learning approach. High prediction performance is achieved through the proposed approach compared to data balancing techniques.

Bon-Gang Hwang.et.al. [15] proposed a knowledge based decision support system to identify the decision factors for prefabricated prefinished volumetric construction. Based on the access feasibility and adoption factors these decision support system is developed to attain better decisions to implement prefabricated prefinished volumetric construction in Singapore. From the above survey it is observed most of the decision support system are processed through conventional statistical models. Very less research works are processes through machine learning models and the efficiency of the system could be improved further. Considering the above issues, the proposed model is designed as a hybrid approach which efficiently provides decision support to the marketing domain.

\section{Proposed work}

The proposed hybrid model is developed as a combination of decision tree and artificial neural network model. This section provides the necessary mathematical formulation for the proposed hybrid algorithm. The proposed Hybrid model is applied in a manufacturing company to provide a decision towards their marketing ideas considering the issues present in the company and its marketing section. The choice of retained company which leads into a poor image in the environment and stack holders, so it is essential to obtain a decision support system to development the production and marketing strategies, so that the company profit could increase. In order to assess the actual situation different pressures are considered which increases the strategical benefits. A list consist of all the necessary criteria is designed and sent to the expert team and marketing team. Reponses from the experts and marketing team are analysed further to obtain the necessary sub-criteria for the proposed system. Based on the analysis again separate list is generated and it is communicated to everyone in the team to obtain the feedback over the selected criteria. In the last, the consensus is selected based on the sub-criteria which is used to refine the industry production and marketing process. process flow of the proposed hybrid model is depicted in figure 3, which has three phased of operation as problem setting, model development, action developments.

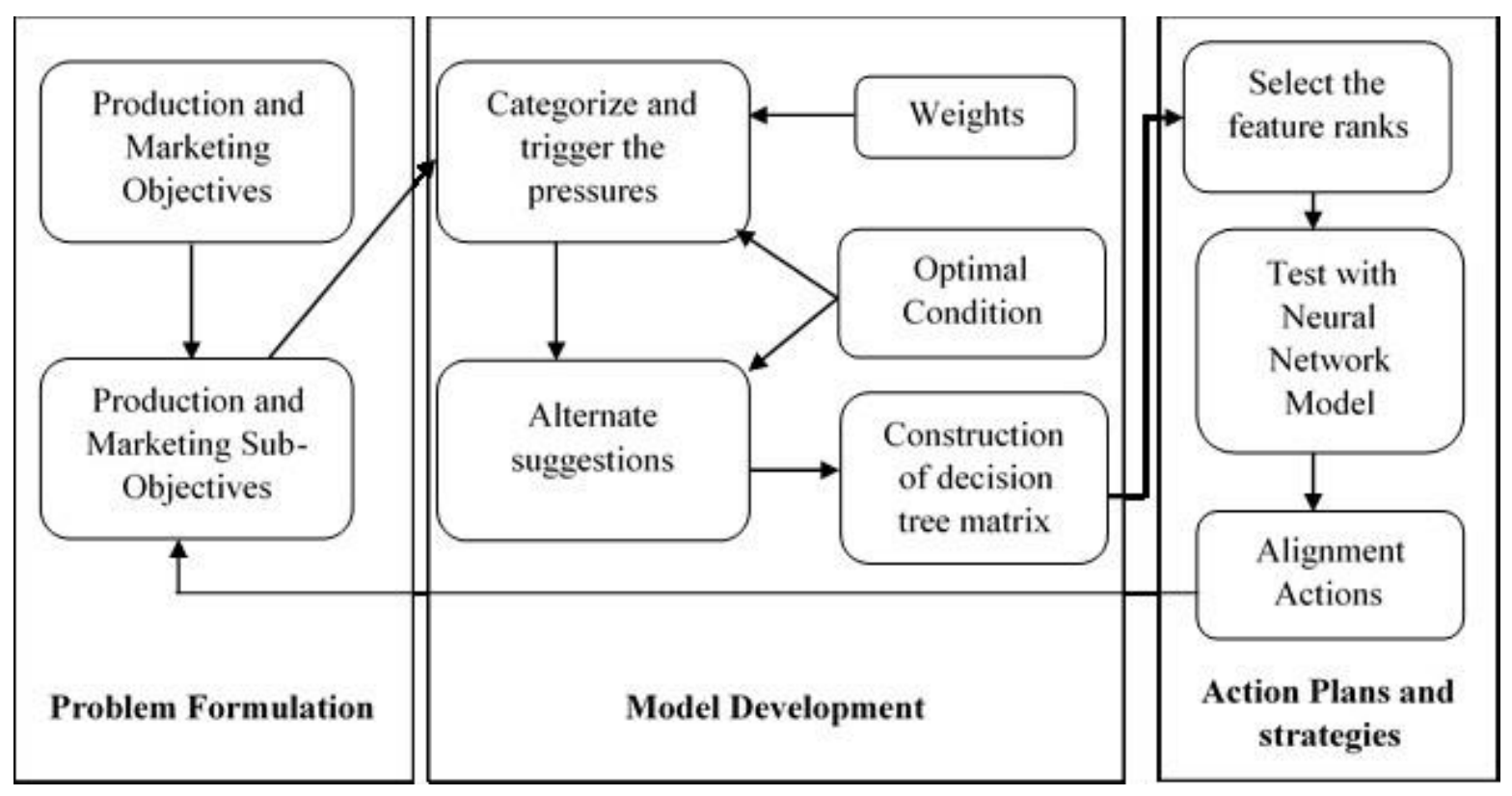

Fig.3 Proposed Hybrid Model

The initial problem formulation phase is used to define the scope and identifying the unavoidable issues in the industry. The route-cause analysis and its relationship is essential for decision support system, in order to identify the root a framework is setup for production and marketing as objectives. In this level, the essential 
Vol.02/ No. 03

Pages: $185-193$

http://irojournals.com/aicn/

DOI: https://doi.org/10.36548/jaicn.2020.3.007

production and marketing objectives are further subdivided in order to reach the framework from top level to bottom level employees. Based on hierarchical order, these objective functions are created.

Model development is the second phase which includes formal models. These models are developed based on the sub-objectives, so that the present manufacturing and marketing aspects could be rearranged. Considering the normalizing, constituting and decision tree weight functions which is given as a matrix function

$\underset{n \times m}{D=}[D]$

$i j$

where the column of the matrix is used to represent the alternate solutions and row of a matrix is used to represent the triggered pressures. The final expanded matrix function for the given objective is $D_{11} \ldots . \quad D_{1 j} \ldots . \quad D_{1 m}$

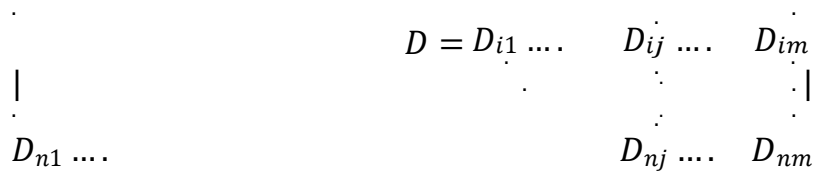

The Normalized decision matrix is given as

$$
D_{N}=\left[D^{\prime}{ }_{i j}\right]_{n \times m}
$$

where $D^{\prime} \underset{\substack{i \sqrt{n} k=1 \\ k}}{D_{, k j}}, i=1,2,3 \ldots . n, j=1,2,3 \ldots . . m$.

Artificial neural network is used to refine the results of decision tree model. Figure 4 depicts the neural network model used in the proposed decision support system for classifying the decision tree hierarchical results.

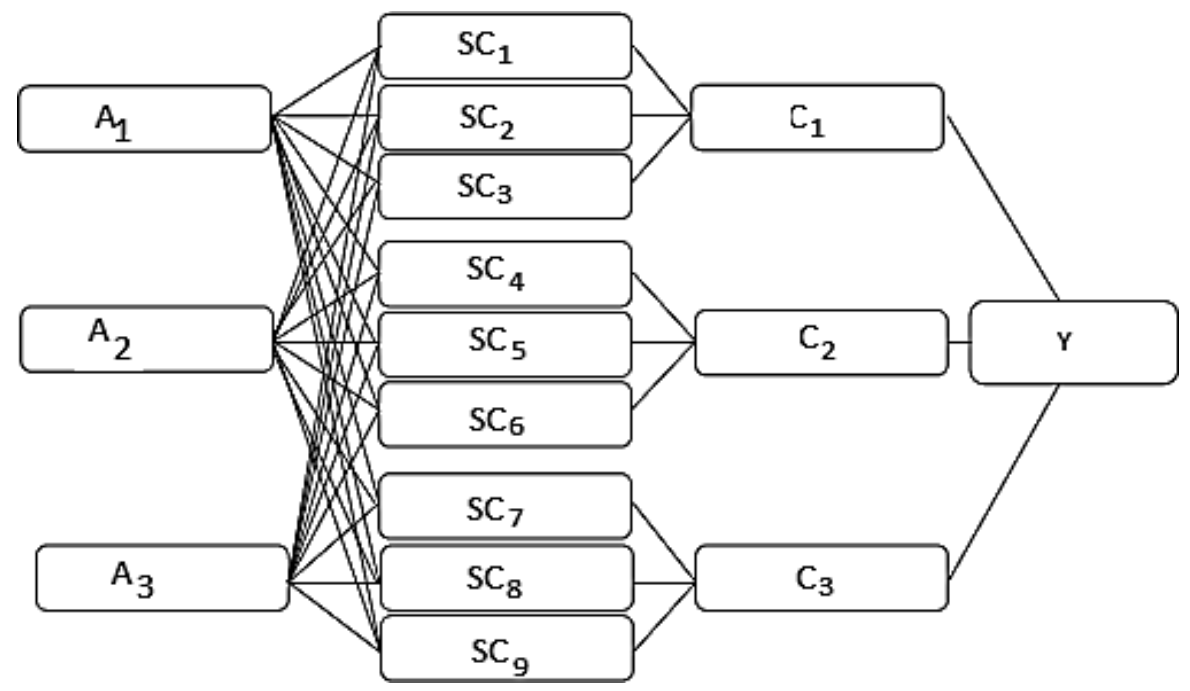

Fig.4 Neural Network Model

The process starts from the three level inputs from the decision tree system and then it is processed into 9 different intermediate level based on the sub-objectives defined for the proposed decision support system. The interlinked process indicates the proposed system has interaction with top level to bottom level of employee in an organization. Based on the sub-objective it is cumulated and classified to obtain the final strategical plan for the manufacturing and marketing process.

ISSN: 2582-2012 (online)

Submitted: 29.07 .2020

Accepted: 20.08.2020

Published: 28.08.2020 
Vol.02/ No. 03

Pages: $185-193$

http://irojournals.com/aicn/

DOI: https://doi.org/10.36548/jaicn.2020.3.007

\section{Result and Discussion}

The proposed hybrid model is analysed based on the criteria such as internal pressure, criteria, targets and external pressures. So that each category is evaluated against the proposed algorithm and compared with conventional hidden Markov and support vector machine based decision support systems. Main objectives are given as $\mathrm{A}_{1}$ for internal pressure, $\mathrm{B}_{1}$ for external pressures and $\mathrm{C}_{1}$ for target and production criteria, where the subobjectives are given as $\mathrm{SC}_{1}$ for job satisfaction (Common for production and marketing employees), $\mathrm{SC}_{2}$ for cognitive skills, $\mathrm{SC}_{3}$ for employee contribution to organization, $\mathrm{SC}_{4}$ for employee power, $\mathrm{SC}_{5}$ for individual efforts, $\mathrm{SC}_{6}$ for timely response, $\mathrm{SC}_{7}$ for available resource, $\mathrm{SC}_{8}$ for organization culture, $\mathrm{SC}_{9}$ for maturity towards company activities. The classification results are clustered into $\mathrm{C}_{1}, \mathrm{C}_{2}, \mathrm{C}_{3}$ and then final classification results are obtained as output of decision support system for organization growth.

Table 1. Decision Tree Matrix

\begin{tabular}{|c|c|c|c|c|c|c|c|c|c|}
\hline & $\mathrm{SC}_{1}$ & $\mathrm{SC}_{2}$ & $\mathrm{SC}_{3}$ & $\mathrm{SC}_{4}$ & $\mathrm{SC}_{5}$ & $\mathrm{SC}_{6}$ & $\mathrm{SC}_{7}$ & $\mathrm{SC}_{8}$ & $\mathrm{SC}_{9}$ \\
\hline $\mathrm{A}_{1}$ & 2 & 2 & 2 & 4 & 4 & 4 & 8 & 5 & 5 \\
\hline $\mathrm{B}_{1}$ & 5 & 5 & 4 & 5 & 5 & 5 & 6 & 3 & 7 \\
\hline $\mathrm{C}_{1}$ & 6 & 8 & 7 & 6 & 5 & 6 & 7 & 8 & 6 \\
\hline
\end{tabular}

Table 1 provides details about decision tree matrix values for the clusters with respect to the main objectives and sub-objectives. It is observed that the external pressure is almost same for all the three categories. On contrary, the internal pressure is much greater for sub-objectives. In terms of individual efforts, the attributes are high compared to others which depicts that the employees are willing to contribute their skills to improve the organization as a team and an individual.

Table 2 Clustering Comparison for the Criteria

\begin{tabular}{|c|c|c|c|c|c|c|c|c|c|c|c|}
\hline & \multicolumn{3}{|c|}{$\mathrm{C}_{1}$} & \multicolumn{3}{|c|}{$\mathrm{C}_{2}$} & \multicolumn{3}{|c|}{$\mathrm{C}_{3}$} & \multirow[b]{2}{*}{$\begin{array}{l}\text { Priority } \\
\text { Function } \\
\text { values }\end{array}$} \\
\hline & & $\mathrm{SC}_{1}$ & $\mathrm{SC}_{2}$ & $\mathrm{SC}_{3}$ & $\mathrm{SC}_{4}$ & $\mathrm{SC}_{5}$ & $\mathrm{SC}_{6}$ & $\mathrm{SC}_{7}$ & $\mathrm{SC}_{8}$ & $\mathrm{SC}_{9}$ & \\
\hline \multirow{3}{*}{$\mathrm{C}_{1}$} & $\mathrm{SC}_{1}$ & 1 & 1 & 2 & 3 & 4 & 2 & 4 & 1 & 1 & 0.1654 \\
\hline & $\mathrm{SC}_{2}$ & 1 & 1 & 2 & 3 & 4 & 2 & 3 & 1 & 1 & 0.1654 \\
\hline & $\mathrm{SC}_{3}$ & 1 & 2 & 3 & 1 & 4 & 1 & 2 & 1 & 2 & 0.1452 \\
\hline \multirow{3}{*}{$\mathrm{C}_{2}$} & $\mathrm{SC}_{4}$ & $1 / 2$ & $1 / 2$ & 1 & 3 & 3 & 2 & 1 & 3 & 3 & 0.0564 \\
\hline & $\mathrm{SC}_{5}$ & $1 / 3$ & $1 / 2$ & $1 / 3$ & 1 & 2 & 2 & $1 / 4$ & $1 / 4$ & $1 / 2$ & 0.0562 \\
\hline & $\mathrm{SC}_{6}$ & $1 / 4$ & $1 / 4$ & $1 / 3$ & 1 & 2 & 2 & $1 / 2$ & $1 / 2$ & $1 / 4$ & 0.0456 \\
\hline \multirow{3}{*}{$\mathrm{C}_{3}$} & $\mathrm{SC}_{7}$ & $1 / 3$ & $1 / 3$ & $1 / 3$ & 2 & 3 & 3 & $1 / 4$ & 1 & $1 / 4$ & 0.0445 \\
\hline & $\mathrm{SC}_{8}$ & $1 / 2$ & $1 / 2$ & 1 & 4 & 2 & 1 & 1 & $1 / 4$ & 4 & 0.0812 \\
\hline & $\mathrm{SC}_{9}$ & $1 / 2$ & 1 & $1 / 2$ & 4 & 2 & 1 & $1 / 4$ & $1 / 2$ & 1 & 0.1256 \\
\hline
\end{tabular}

Table 2 depicts the clustering comparison details for the criteria and it is built based on the response of objectives and sub-objectives from managers to low level employees. The priority function value is obtained based on the consistency of the clustering and threshold values. It is acceptable if the value is below 0.1 and others are considered as non-acceptable. 


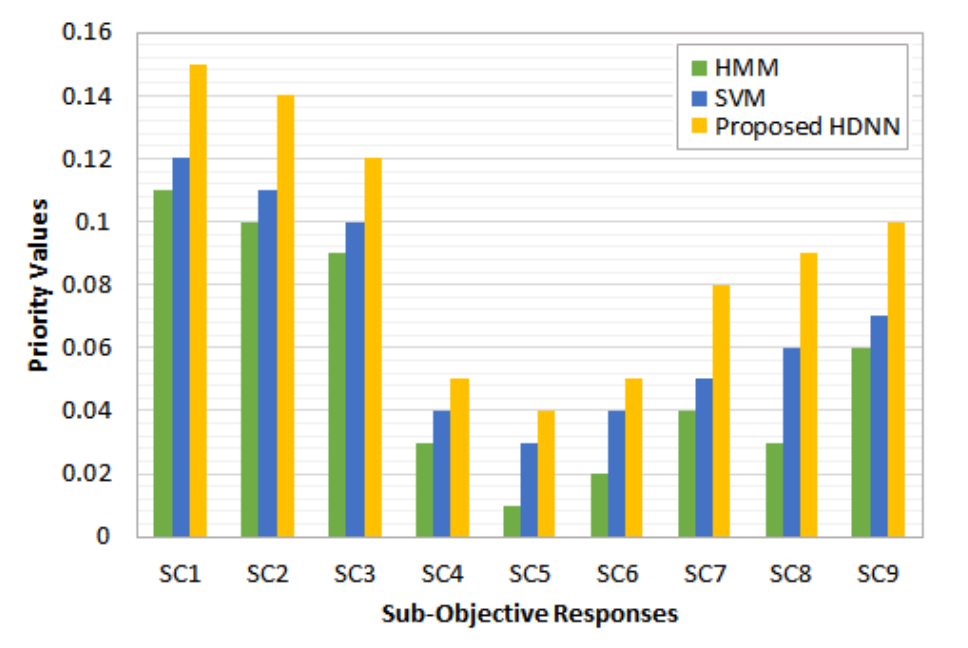

Fig. 5 Priority assessment comparison

Based on the weights the priority values of the proposed system are measured and compared with hidden Markov model and support vector model and it is depicted in figure 5. It is observed that proposed model has better priority values by assessing the employees, stack holders and management opinions. While the other models have less priority values due to its inefficient assessment strategies.

\section{Conclusion}

A hybrid decision support system is proposed in this research work using decision tree and artificial neural network. The issues in existing decision support models are identified to develop this decision support system to improve the manufacturing and marketing strategies of an organization. Based on the option obtained from the employees, the main objective function is subdivided into sub-objective function to analyse the organization from top level to bottom level. Given responses are analysed using decision tree matrix and trained using artificial neural network to obtain better classification strategies. Experimental results are observed and compared with conventional hidden Markov Model and support vector based decision support system. Proposed model attains better classification results compared to other model which could be applicable to investigate various domains. Further this research work could be improved by including an optimization models to improve the assessment performance.

\section{References}

1. Daniel Bumblauskas, Douglas Gemmill, Amy Igou, Johanna Anzengruber (2017). Smart Maintenance Decision Support Systems (SMDSS) based on corporate big data analytics. Expert Systems with Applications.90: 303-317.

2. Sebastian Abele, Michael Weyrich (2017). Decision Support for Joint Test and Diagnosis of Production Systems based on a Concept of Shared Knowledge. IFAC-PapersOnLine. 50(1): 15227-15232.

3. İhsan Erozan (2019). A fuzzy decision support system for managing maintenance activities of critical components in manufacturing systems. Journal of Manufacturing Systems. 52:110-120.

4. Francesco Lolli, Alessio Ishizaka, Rita Gamberini, Bianca Rimini, Laura Prandini (2017). Requalifying public buildings and utilities using a group decision support system. Journal of Cleaner Production.164: 1081-1092. 
5. Atiq W. Siddiqui, Syed Arshad Raza, Zeeshan Muhammad Tariq (2018). A web-based group decision support system for academic term preparation. Decision Support Systems. 114:1-17.

6. Morteza Yazdani, Pascale Zarate, Adama Coulibaly, Edmundas Kazimieras Zavadskas (2017). A group decision making support system in logistics and supply chain management. Expert Systems with Applications. 88: $376-392$.

7. Katia Regina Evaristo de Jesus, Sérgio Alves Torquato, Pedro Gerber Machado, Catiana Regina BrumattiZorzo, Dilvan A. Moreira (2019). Sustainability assessment of sugarcane production systems: SustenAgro Decision Support System. Environmental Development. 32:1-15.

8. Nicholas J. Car (2018). USING decision models to enable better irrigation Decision Support Systems. Computers and Electronics in Agriculture.152:290-301.

9. Yang Luo, John Andresen, Henry Clarke, Matthew Rajendra, Mercedes Maroto-Valer (2019). A decision support system for waste heat recovery and energy efficiency improvement in data centres. Applied Energy, 250: 12171224.

10. Mahsa Dehghani Soufi, Taha Samad-Soltani, Samad Shams Vahdati, Peyman Rezaei-Hachesu (2018). Decision support system for triage management: A hybrid approach using rule-based reasoning and fuzzy logic. International Journal of Medical Informatics. 114:35-44.

11. Ahmed S. Negm, Osama A. Hassan, Ahmed H. Kandil (2018). A decision support system for Acute Leukaemia classification based on digital microscopic images. Alexandria Engineering Journal.57(4): 2319-2332.

12. Marieke Pereboom, Inge J. Mulder, Sjoerd L. Verweij, Ruud T. M. van der Hoeven, Matthijs L. Becker (2019). A clinical decision support system to improve adequate dosing of gentamicin and vancomycin. International Journal of Medical Informatics. 124:1-5.

13. Muhammad Fadzli Ali, Ammar Abdul Aziz, Siti Hawa Sulong (2020). The role of decision support systems in smallholder rubber production: Applications, limitations and future directions. Computers and Electronics in Agriculture. 173:1-14.

14. Zouhair Elamrani Abou Elassad, Hajar Mousannif, Hassan Al Moatassime (2020). A proactive decision support system for predicting traffic crash events: A critical analysis of imbalanced class distribution. Knowledge-Based Systems. 205:1-14.

15. Bon-Gang Hwang, Ming Shan, Kit-Ying Looi (2018). Knowledge-based decision support system for prefabricated prefinished volumetric construction. Automation in Construction. 94:168-178. 